\title{
Laryngeal Transplantation
}

\author{
Review $>\begin{aligned} & \text { Öner Sakallığlu } \\ & \text { Clinic of Otorhinolaryngology, Elazığ Training and Research Hospital, Elazığ, Turkey }\end{aligned}$
}

Abstract Patients who require laryngectomy usually do not want to completely or partially lose their larynx. Laryngeal transplantation (LT) is a composite tissue transplantation from a cadaver to an alive recipient and requires lifelong immunosuppression in postoperative term. The aims of LT are breathing without tracheostoma, normal swallowing, and voice production. To date, only two successful complete LT have been performed in human de-

\section{Introduction}

Organ transplantation is the transfer of a partial or complete healthy organ from an organ donor to replace the damaged or absent organ of a recipient. The organ donor may be a living person or a cadaver. Laryngeal transplantation (LT) is composite tissue transplantation. Composite tissue transplantations are operations performed to heal patients with composite tissue loss that impairs body integrity and causes dysfunction, thus improving their life quality.

LTs involve a short absence of blood circulation and the possibility of rejection by the recipient; they also require venous anastomosis and reinnervation, as well as immunosuppressive therapies (1, 2). Although organ transplantation is rapidly developing, it is one of the most controversial issues in modern medicine. Despite the advanced practices in this area (such as heart, kidney, and liver transplantation), transplantations of some organs, including the face, arm, and hand, are still new.

Moreover, some patients who have esthetic and functional problems with laryngectomy can also undergo LT, despite the necessity for immunosuppressive treatment for the rest of their lives (4). In other words, LT can be an alternative for improving life quality in patients undergoing laryngectomy (5). Patients with the indication of laryngectomy usually do not want to lose their larynxes partially or completely. McNeil et al. (3) reported that when patients who were offered choices of radiotherapy or laryngectomy were told that they had a higher chance of survival with laryngectomy, $20 \%$ of the patients preferred radiotherapy. Potter and Brichall (4) conducted a study where they surveyed members of the National Association of spite many researches. The requirement of post-transplant immunosuppressive treatment has ethical concern for the larynx, which is a non-vital organ. However, LT may be an option for improving the quality of life of patients undergoing laryngectomy. In this study, we discussed the LT procedure and researches with the literature.

Keywords: Laryngectomy, larynx, transplantation, airway reconstruction, immunosuppression

Laryngectomy Clubs; they found that $75 \%$ of patients would accept LT under ideal conditions and that most patients would prefer LT of laryngectomy to a life without the larynx. It was observed that younger patients' attitudes toward LT were more positive. In a study conducted by Buiret et al. (6) on laryngectomy patients in L'Association Nationale des Mutiles de la Voix, it was found that $63 \%$ of patients (most younger than 59 years) found LT acceptable despite its medical limitations (duration of hospitalization of at least one year and monitoring by the transplant center for years) and functional risks (permanent cannula, unguaranteed laryngeal sound, or unguaranteed functional swallowing). Monaco (7), a doctor studying organ transplantation, also underwent laryngectomy; she stated in her article that "if I were 40 years old I would probably consider undergoing the operation myself."

The concept of LT in human beings began with studies conducted on animal models by Boles, Ogura, and Silver (8). The first LT in a human being was performed by Kluykens and Ringoir (9); the tissue was transplanted from a 40-year-old cadaver to a 62-year-old patient with laryngeal cancer on February 10,1969 . This transplantation was performed by protecting the perichondrium of the recipient and maintaining the subtotality and revascularization of the donor organ. Because patients were lost due to rapid tumor recurrence (8 months after operation), studies on LT were stopped for approximately 20 years (8). In 1987, Strome began to study total LT and suggested four important concepts for successful transplantation. These include revascularization, reinnervation, rejection, and ethical concerns that can result from the transplantation of the larynx, which is a non-vital organ. Finally, the first total LT

\author{
E-mail: onersakallioglu@yahoo.com \\ Received Date: 09.03.2015 \\ (c) Copyright 2015 by Official Journal of the Turkish \\ Society of Otorhinolaryngology and Head and \\ Neck Surgery Available online at \\ www.turkarchotorhinolaryngol.org \\ DOl: $10.5152 / \operatorname{ta0} .2015 .999$
}


in a human being was performed by Marshall Strome on January 4, 1998 (8). Although LT case series have been reported from Colombia, there is no clear information regarding their results and techniques (10).

The ultimate goal of LT is to provide respiration without tracheostomy, normal swallowing, and sound production. The larynx transplanted for this aim must provide adequate vocal cord adduction, abduction (for respiration and sound), and laryngeal elevation (for swallowing). Moreover, to prevent aspiration, the sensory function must be obtained at the level at which it can be recognized that food and secretions are in the larynx. The quality of sound that is produced by a transplanted larynx is not obviously different from the patient's previous quality of sound. Because the accent of the patient is formed by the lips, cheek, tongue, and palate (articulators), it must be expected that the accent will not greatly change. On the other hand, fine motor control is necessary for obtaining prosodic features including speech rhythm, stress, and tonation (11).

It is important to assess a patient who is thought to be a candidate for LT from a psychosocial aspect. To reduce risks related to an impaired immune system, the patient should understand the lifelong immunosuppression risk. In addition, the patient's compliance to postoperative drug therapy and changes in his/ her ongoing behavioral patterns should be carefully checked (11). To date, laryngeal, tracheal, and facial transplantations in the head-neck region have been successfully performed. Laryngeal and facial transplantations are vascularized composite tissue transplantations, and they require immunosuppression. Immunosuppressive therapies carry an important risk for secondary malignancy as well as nephrotoxic and hepatotoxic effects. The transplantation of a non-vital organ, such as the larynx, in patients with head and neck cancer will increase the risks of cancer recurrence or secondary malignancy (12). Therefore, the fact that the larynx requires immunosuppressive treatment after transplantation can create concern from an ethical perspective (13).

\section{Clinical and Research Effects}

The first successful total LT in a human being was performed by Marshall Strome and his team at the Cleveland Clinic on January 4, 1998 (Cleveland, Ohio, USA).

\section{The First Total LT Patient and Donor}

The first total LT was performed on a 40-year-old aphonic patient with a damaged larynx and pharynx due to a motorcycle accident that occurred 20 years previously (Timothy Heidler). The patient underwent a permanent tracheotomy and also experienced losses in taste and smell perception. The patient used an electrolarynx for speaking. A donor larynx was found 6 months later. The donor was a 40-year-old non-smoker who died due to a ruptured cerebral aneurysm and who did not have any other diseases (14). The entire pharyngolaryngeal complex, including six tracheal rings as well as the thyroid and parotid glands, was removed with related vascular structures from the donor.

\section{Applied Surgical Procedure}

First, the laryngeal vein and nerves were revealed during the operation. The right superior thyroid artery of the donor was anastomosed to that of the patient. The proximal end of the donor's right internal jugular vein was anastomosed to the common facial vein of the patient. The distal end of the donor's jugular vein was sutured to itself. Then a narrow-field laryngectomy was applied to the recipient patient, and the trachea was removed with the upper half of the stoma. The thyroid gland of the recipient was divided and repositioned toward the lateral sides. The hyoid bone of the patient was not altered to contribute to laryngeal elevation. The narrow pharynx of the patient was expanded, and fixation and elevation were provided by placing three permanent sutures between the patient's hyoid bone and the donor's thyroid cartilage. The donor's tracheal rings were sutured on the patient's, and a new stoma was formed at a higher level. The left superior thyroid arteries were anastomosed tip to tip. The left middle thyroid vein of the transplant was anastomosed to the recipient's left internal jugular vein. Both superior laryngeal nerves and the right recurrent nerve of the transplant were sutured to those of the recipient. The left laryngeal nerve of the patient could not be identified due to existing scar tissues.

\section{Postoperative Course}

The tracheostomy tube and drains were removed on the first postoperative day. The existing permanent tracheotomy of the patient was converted into a new stoma by using tracheal rings transplanted from the donor during the operation; thus, it could be decannulated in the early postoperative period. Furthermore, direct postoperative application of glycopyrrolate and atropine decreased salivary secretion and provided early decanulation. Until he learned to swallow, the patient was fed by gastrostomy for 14 weeks. One week after the operation, morumonab, cyclosporine, methylprednisolone, and mycophenolate mofetil were given. The first rejection attack was observed in the $15^{\text {th }}$ postoperative month. This attack was controlled with a high dose of prednisone (14). The second rejection attack occurred six years after LT, in 2003.

Sound production was impaired, and an endoscopy revealed edema in the epiglottis and glottis. Improvement was obtained by supplying intravenous salmeterol (15). On the third postoperative day, the patient said his first word ("hello") in 20 years. Electromyography (EMG) confirmed that both the vocal cords and cricothyroid muscles were reinnervated. In the subjective and objective evaluation of phonation 16 weeks after LT, it was found to be near normal. In the $36^{\text {th }}$ month, speech characteristics were within normal intervals. In the third postoperative month, the supraglottis and vocal cords became sensitive to touch, and a cough reflex was observed. No solid and fluid aspiration was observed in the barium swallow test. The taste and smell perception of the patient were regained. The serum calcium, phosphate, and thyroid hormone levels of the patient were found to be normal (14). 


\section{The Second Total LT Patient and Donor}

The second LT was performed by a team directed by Gregory Farwell at the University of California (UC) Davis Medical Center on October 13, 2010 (Sacramento, California, USA). A 52-year-old female patient (Brenda Charett Jensen) underwent laryngeal, thyroid gland, and tracheal transplantation. Before the transplantation, Brenda could not speak or breathe normally, and her airway was affected due to previous operations. She was completely dependent on permanent tracheotomy. She used an electrolarynx for speaking. A person who died at the age of 38 years due to anoxic arrest, who did not have any other health problems, was chosen as the donor. The laryngoscopy result was evaluated to be normal.

\section{Applied Surgical Procedure}

A 6-cm segment was removed from the larynx, thyroid gland, and trachea of the donor. The donor's larynx, thyroid, parathyroid glands, and large supply veins, including the supraaortic truncus, were removed. The superior and inferior laryngeal nerves were removed as they were isolated from the surrounding tissues. The recipient underwent a narrow field laryngectomy in preparation for transplantation. The distal trachea was not removed so that a proper tracheostomy might be applied in the event that the transplantation failed (16).

The patient's right superior thyroid artery, bilateral transverse cervical arteries, and bilateral internal jugular veins were prepared for microanastomosis. The diameter of the left superior thyroid artery was approximately $1 \mathrm{~mm}$, and it was determined to be too small for anastomosis. The thyroid gland was divided and adjusted at the level of the inferior thyroid artery. Both superior laryngeal nerves, the recurrent laryngeal nerves, the left phrenic nerve, and the ansa cervicalis were isolated. First of all, the donor's right superior thyroid artery was anastomosed end-laterally to the patient's right superior artery, and the donor's right brachiocephalic vein was anastomosed end-laterally to the recipient's right inferior jugular vein. Then, on the right side, the transverse artery of the recipient was anastomosed to the donor's inferior thyroid artery. The bilateral superior and recurrent laryngeal nerves of the donor and patient were anastomosed with microneurography. The donor's thyroid cartilage was sutured to the patient's hyoid bone with 2-0 polypropylene material to provide laryngeal suspension (16). Finally, an end-lateral anastomosis was made between the donor's left recurrent laryngeal nerve and the patient's left phrenic nerve. After the recipient's larynx was removed, eight tracheal rings were anastomosed with the larynx while preventing the occurrence of tension.

\section{Postoperative Course}

The patient underwent rehabilitation for 2 months after the operation, which lasted 18 hours. Brenda re-learned to speak and swallow after LT. She regained her perceptions of taste and smell after many years. Brenda's voice was similar to her own, not that of the donor. Because Brenda had undergone a kidney-pancreas transplantation four years previously (in 2006), and thus was a transplant patient, she was already receiving immunosuppressive therapy with tacrolimus and leflunomide. For LT immunosuppression, anti-thymocyte globulin (rabbit), methylprednisolone, tacrolimus, and mikofenolate were initiated. Rejection attack was not observed in the patient. Brenda remained in the hospital for four weeks after LT. On the 13th postoperative day (October $29,2010)$, the patient began to say her first words in 11 years. Brenda said that this operation restored her life; she felt lucky to have this opportunity, and she was thankful to the donor and the family. The sound that she produced at first was similar to a caw, but understandable, which made her smile. When she was discharged from the hospital on November 11,2010, the state of her health was quite good (17).

\section{Immunosuppressive Therapy After LT}

At present, one of the most important problems related to LT is immunosuppressive treatment. Lifelong immunosuppression is applied after all transplantation procedures. Current immunosuppressive protocols can provide a rate of graft viability above $90 \%$ within the first year after the transplantation. Studies have shown that rejection can be efficiently prevented by a regime of alemtuzumab (a single dose on the day of transplantation) and tacrolimus (long term), and long-term viability of the transplanted larynx can be enabled.

\section{Hemilaryngeal Transplantation}

Andrews et al. (18) emphasized that there was no ideal method for the reconstruction of hemilaryngeal defects because any autologous flap or graft cannot replace the unique structural features of the larynx; therefore, they performed hemilaryngeal transplantation in the canine model. According to Andrews et al. (18), optimal phonation occurs when both vocal cords have similar muscle and mucosal features. As a result of the reconstruction of hemilaryngeal defects, the residual vocal cord vibrates the opposite pseudo-cord formed by the mucosa, cartilage, or myofascial flaps. In this way, an adequate airway can be obtained and aspiration can be prevented; however, phonation cannot be produced sufficiently.

The procedure of hemilaryngeal transplantation in the canine model by Andrews et al. (18), briefly, consisted of reinnervating the donor's thyroarytenoid muscle using the anterior branch of the recurrent laryngeal nerve and providing arytenoid adduction. After hemilaryngeal transplantation, immunosuppressive treatment was given, including azathioprine, cyclosporine and prednisone.

The details of the technique were as follows. First, the recipient animal was prepared. In the left side, the true vocal cord, ventricle, false vocal cord, intrinsic muscles, arytenoid cartilage, and anterior $2 / 3$ of the thyroid cartilage were removed. In the donor animal, the hyoid venous arcus and the external jugular vein in the left side were protected for the graft to provide venous drainage. The cranial thyroid artery, which is a branch of the 
common carotid artery, was also protected. The thyroarytenoid branch of the recurrent laryngeal nerve was identified, marked, and excised. As defined, mucosal, muscle, and cartilage incisions were performed. The donor's left hemilarynx was ligated from the superior and inferior cranial thyroid branches of the common carotid artery and removed. The external jugular vein was ligated from the distal venous branches of the hyoid arcus and removed. Then the donor's hemilarynx was placed into the recipient's bed, and arterial circulation was provided through endto-end anastomosis of the common carotid artery. After that, the external jugular veins were anastomosed end-to-end. The recipient's recurrent laryngeal nerve anterior branch was anastomosed to the donor's thyroarytenoid branch. Other intrinsic laryngeal muscles were not reinnervated. Arytenoid adduction was provided by fixating the donor's arytenoid to the recipient's cricoid. Internal mucosal and external perichondrial sutures were then used for closing. In the stimulated EMG, the transplanted thyroarytenoid muscle was observed to be contracted due to stimulation (18).

Andrews et al. (18) reported that hemilaryngeal transplantation was a good example of partial organ transplantation, and results comparable with the performance of known techniques could be obtained for vertical hemilaryngectomy reconstruction. They emphasized that the most important limitation of LT in human beings was cancer recurrence, second primary cancer, or infection-induced complications due to immunosuppressive treatment.

\section{Related to Donors}

It has been reported that studies on laryngotracheal transplantation in human beings have been performed in Colombia since 2002. In a study in which 25 larynx and trachea donors were examined in Colombia, the organs of five donors were designated for further study; seven were not used because their laryngoscopic examinations were abnormal. Thirteen donors were evaluated to be suitable for transplantation, and 13 laryngotracheal transplantations were performed. The mean age of these 13 donors was $27.2 \pm 7.9$ years, and all of them died from head trauma. All were male, and 12 were multiorgan donors. Three donors had used tobacco, and two donors had used marijuana. One patient had a history of asthma. Rejection attack was observed in two patients, and the graft viability rate was approximately $90 \%$ over two years. Only one patient died due to a stroke that occurred during the operation (10).

Duque et al. (10) defined the criteria that must be used for the identification of appropriate donors as follows:

1) Age between 18 and 50 years

2) Gender and $\mathrm{ABO}$ blood type match between the donor and recipient

3) No use of tobacco, cocaine, cocaine paste, or marijuana

4) A history of tracheal intubation shorter than three days

5) Length of stay in the intensive care unit shorter than seven days

\section{Negative Views about LT}

Birchall and Macchiarini (19) stated that LT was performed in the human body only once, although studies on LT had been conducted for approximately 40 years. They pointed out that some points, such as the success rate of reinnervation, the cost of the procedure, and immunosuppressive treatment, must be considered before beginning new detailed clinical studies. Moreover, it was stated that although patients who underwent laryngectomy due to cancer were the primary targets, immunosuppression involved high risk for these patients and that some ethical problems could occur.

\section{Conclusion}

There are many ongoing experimental studies on LT. The results for patients having undergone LT are highly encouraging. Studies have revealed that patients who have undergone total laryngectomy due to trauma and benign or low-staged malignant diseases are proper candidates for LT (11). It is assumed that patients with advanced local diseases or recurrent laryngeal malignancy are not proper candidates for LT due to the high risk of the development of secondary cancer. The most restrictive factors for LT may be non-immunological side effects and chronic immunosuppressive therapies that can negatively affect cancer rates (20). Because LT can be necessary for patients having undergone total laryngectomy due to advanced stage cancer, immunosuppressive therapies that do not increase malignancy potential should be developed in the future. In addition, despite success at various rates, further studies should be performed on selective reinnervation in the larynx that will be transplanted. Because all functions of the larynx cannot be regained with conventional reconstruction techniques and prostheses, increasing life quality through LT is important in patients with irreversible laryngeal disease. Successful implementation of solid organ transplantation, such as the heart, liver, and kidney, has contributed to improvement in immunological and surgical techniques. Moreover, the fact that recent arm and face transplantations can be performed without a need for intense immunosuppressive therapy suggests that the same successful implementation also occur in LT. While knowledge on LT is increasing daily, there are still many questions to be answered. For this purpose, more comprehensive clinical and laboratory studies should be conducted. In conclusion, the transplantations of organs that increase life quality as well as of vital organs should be made acceptable to patients.

Peer-review: Externally peer-reviewed.

Acknowledgements: I thank to Prof. Dr. Ahmet Ömer İkiz, Assoc. Prof. Dr. Görkem Eskiizmir and Assoc. Prof. Dr. Nilda Süslü who are the educators of School of Head and Neck and Thyroid Surgery in 2014-2015 training season.

Conflict of Interest: No conflict of interest was declared by the author.

Financial Disclosure: The author declared that this study has received no financial support. 


\section{References}

1. Kececioglu N, Ozkan O, Ozkan O. Kompozit doku nakli. Türkiye Organ Nakli Vakfi. (cited 2014 January 15). Available from: http://tonv.org.tr/tr/proje/kompozit-doku-nakli/7/0

2. Ayhan S, Tugay C, Porvasnik S, Crownover R, Siemionow M. Effect of low-dose donor radiation on acute rejection of composite limb allografts. Transplant Proc 2000; 32: 588-90. [CrossRef]

3. McNeil BJ, Weichselbaum R, Pauker SG. Speech and survival: tradeoffs between quality and quantity of life in laryngeal cancer. N Engl J Med 1981; 305: 982-7. [CrossRef]

4. Potter CP, Birchall MA. Laryngectomees' views on laryngeal transplantation. Transpl Int 1998; 11: 433-8. [CrossRef]

5. Khariwala SS, Lorenz RR, Strome M. Laryngeal transplantation: research, clinical experience, and future goals. Semin Plast Surg 2007; 21: 234-41. [CrossRef]

6. Buiret G, Rabilloud M, Combe C, Paliot H, Disant F, Céruse P. Larynx transplantation: laryngectomees' opinion poll. Transplantation. 2007; 84: 1584-9. [CrossRef]

7. Monaco AP. Transplantation of the larynx--a case report that speaks for itself. N Engl J Med 2001; 344: 1712-4. [CrossRef]

8. Lorenz RR, Fritz MA, Strome M. Special feature: ccurrent state of laryngeal transplantation. Curr Opin Otolaryngol Head Neck Surg 2001; 9: 381-6. [CrossRef]

9. Kluyskens P, Ringoir S. Follow-up of a human larynx transplantation. Laryngoscope 1970; 80: 1244-50. [CrossRef]

10. Duque E, Duque J, Nieves M, Mejía G, López B, Tintinago L. Management of larynx and trachea donors. Transplant Proc 2007; 39: 2076-8. [CrossRef]

11. Narula T, Bradley P, Carding P, Hakim N, Rumsey N, Sokol N. Laryngeal transplantation. Working party final report. London
(UK). Department of Otolaryngology, Head and Neck Surgery. The Royal College of Surgeons of England. Advancing Surgical Standarts: 2011 June report. No: 212808.

12. Lou Q, Farwell G. Laryngeal transplantation. Curr Otorhinolaryngol Rep 2014; 2: 192-5. [CrossRef]

13. Lott DG. What is the future of 'organ transplantation' in the head and neck? Curr Opin Otolaryngol Head Neck Surg 2014; 22: 429-35. [CrossRef]

14. Strome M, Stein J, Esclamado R, Hicks D, Lorenz RR, Braun W, et al. Laryngeal transplantation and 40-month follow-up. N Engl J Med 2001; 344: 1676-9. [CrossRef]

15. Knott PD, Hicks D, Braun W, Strome M. A 12-year perspective on the world's first total laryngeal transplant. Transplantation 2011; 91: 804-5. [CrossRef]

16. Farwell DG, Birchall MA, Macchiarini P, Luu QC, de Mattos AM, Gallay BJ, et al. Laryngotracheal transplantation: technical modifications and functional outcomes. Laryngoscope 2013; 123 : 2502-8. [CrossRef]

17. Casey C. Extraordinary larynx transplant restores voice, sense of taste, smell to California woman. Sacramento, California, USA. School of medicine, Betty Irene Moore School of Nursing. News from UC Davis Health System. 2011 January report.

18. Andrews RJ, Berke GS, Blackwell KE, Jakobsen M, Wang MB, Sercarz JA. Hemilaryngeal transplantation in the canine model: technique and implications. Am J Otolaryngol 2000; 21: 85-91. [CrossRef]

19. Birchall M, Macchiarini P. Airway transplantation: a debate worth having? Transplantation 2008; 85: 1075-80. [CrossRef]

20. Nelson M, Fritz M, Lorenz R, Strome M. Update on laryngeal transplantation. Graft 2002; 5: 437-44. [CrossRef] 